\title{
Influence of atmospheric turbulence on states of light carrying orbital angular momentum
}

\author{
Brandon Rodenburg, ${ }^{1, *}$ Martin P. J. Lavery, ${ }^{2}$ Mehul Malik, ${ }^{1}$ Malcolm N. O'Sullivan, ${ }^{1}$ Mohammad Mirhosseini, ${ }^{1}$ \\ David J. Robertson, ${ }^{3}$ Miles Padgett, ${ }^{2}$ and Robert W. Boyd ${ }^{1,4}$ \\ ${ }^{1}$ The Institute of Optics, University of Rochester, 320 Wilmot Building, 275 Hutchison Road, Rochester, New York 14627, USA \\ ${ }^{2}$ School of Physics and Astronomy, University of Glasgow. SUPA, Kelvin Building, Glasgow G12 8QQ, Scotland, UK \\ ${ }^{3}$ Centre for Advanced Instrumentation, Department of Physics, Durham University, Durham, DH1 3LE, UK \\ ${ }^{4}$ Department of Physics, University of Ottawa, Ottawa, Ontario K1N 6N5, Canada \\ *Corresponding author: Brandon.Rodenburg@gmail.com
}

Received May 31, 2012; revised July 24, 2012; accepted July 24, 2012;

posted July 26, 2012 (Doc. ID 169439); published August 31, 2012

\begin{abstract}
We have experimentally studied the degradation of mode purity for light beams carrying orbital angular momentum (OAM) propagating through simulated atmospheric turbulence. The turbulence is modeled as a randomly varying phase aberration, which obeys statistics postulated by Kolmogorov turbulence theory. We introduce this simulated turbulence through the use of a phase-only spatial light modulator. Once the turbulence is introduced, the degradation in mode quality results in crosstalk between OAM modes. We study this crosstalk in OAM for 11 modes, showing that turbulence uniformly degrades the purity of all the modes within this range, irrespective of mode number. (C) 2012 Optical Society of America
\end{abstract}

OCIS codes: $\quad 010.1330,270.5585,270.5565$.

A fundamental concern for any free-space communications channel is the effect that atmospheric turbulence has on the crosstalk between channels. Atmospheric turbulence has been studied at great length by the astronomy community in relation to aberrations in an image [1]. The natural randomly time-dependent variations in temperature and pressure of the atmosphere result in a change in density of the atmosphere. This results in a spatial dependent change of the refractive index leading to a phase distortion across a transmitted beam [2]. A phase distortion of this type can be modeled by a single phase screen, and is commonly referred to as thinphase turbulence [3].

There has been recent interest in the use of spatial modes as an additional degree of freedom to increase the available information bandwidth for free-space communication. One example of these modes are beams carrying orbital angular momentum (OAM). Allen et al. showed that beams with the profile of $\psi_{\ell}=A(r)$ $\exp (i \ell \theta)$ carry an OAM of $\ell \hbar$ per photon [4]. An example of such beams are Laguerre-Gaussian (LG) modes, which have a helical phase structure, with $r$ and $\theta$ as the radial and angular coordinates, respectively. The variable $\ell$ is an unbounded integer, and as such suggests the use of OAM as a variable in free-space optical communication links [ㄷ,ㅎ]. In addition to the advantages of a large alphabet, the security of cryptographic keys transmitted with a quantum key distribution system have been shown to be improved with the use of a large Hilbert space [7].

Recently, there have been several studies on how atmospheric turbulence affects such OAM-based communication [8-12]. In this Letter, we experimentally study the effects of atmospheric turbulence on a communication system utilizing OAM modes as the information carrier. We generate a single OAM mode using a spatial light modulator (SLM). Atmospheric turbulence is then simulated by the addition of a turbulent phase screen to the phase hologram displayed on the SLM shown in Fig. 1(b).
Once the turbulence is applied, the phase aberrations result in a spread of the input mode power over neighboring OAM modes, resulting in crosstalk between the channels. This spread in power is then measured for different turbulence strengths.

(a)

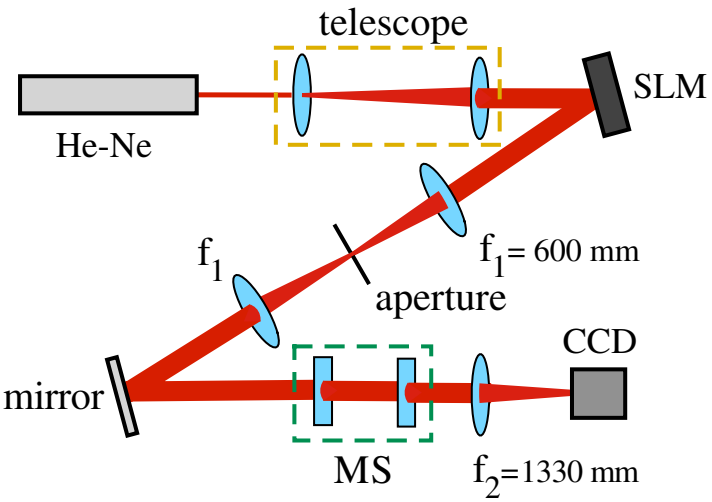

(b)

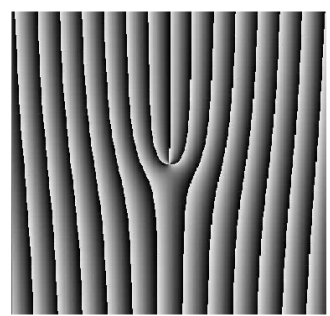

(c)

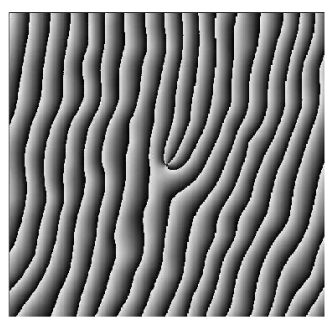

Fig. 1. (Color online) (a) A beam carrying OAM is prepared by the use of an l-forked hologram, seen in (b). This is realized on an SLM illuminated by an expanded HeNe laser. The first order beam is imaged onto the front aperture of an OAM mode sorter (MS), which converts OAM states into transverse momentum states with the use of two refractive optical elements. These transverse momentum states are then focused to specific spatial locations on a CCD. The power measured in each of these locations gives a measure of the OAM superposition incident on the MS. (c) Thin-phase turbulence is added to the $\ell$-forked hologram changing the OAM superposition measured by the system. 
We generate turbulence phase screens according to Kolmogorov turbulence theory [3] . The aberrations introduced by atmospheric turbulence can be considered as normal random variables, where the ensemble average can be written as $\left\langle\left[\phi\left(\mathbf{r}_{1}\right)-\phi\left(\mathbf{r}_{2}\right)\right]^{2}\right\rangle$, which is known as the phase structure function $[\underline{8}, \underline{9}]$. Here, $\phi\left(\mathbf{r}_{1}\right)$ and $\phi\left(\mathbf{r}_{2}\right)$ are two randomly generated phase fluctuations. From Kolmogorov statistics it can be shown that this ensemble average must meet the requirement that

$$
\left\langle\left[\phi\left(\mathbf{r}_{1}\right)-\phi\left(\mathbf{r}_{2}\right)\right]^{2}\right\rangle=6.88\left|\frac{\mathbf{r}_{1}-\mathbf{r}_{2}}{r_{0}}\right|^{5 / 3}
$$

The value $r_{0}$ is the Fried parameter and is a measure of the traverse distance scale over which the refractive index is correlated [3]. To characterize the effect of turbulence on the optical system, the ratio $D / r_{0}$ is considered, where $D$ is the aperture of the system. There are two limiting cases for this ratio: when $D / r_{0}<1$, the resolution of the system is limited by its aperture, and when $D / r_{0}>1$, the atmosphere limits the system's ability to resolve an object [3].

Our theoretical analysis closely follows that of reference [9]. Consider a single OAM mode, $\psi_{\ell}$, transmitted through an ensemble average of many turbulent phase screens. The average detected power, $s_{\Delta}$, in the mode, $\psi_{\ell+\Delta}$, is given by

$$
s_{\Delta}=\frac{1}{\pi} \int_{0}^{1} \rho \mathrm{d} \rho \int_{0}^{2 \pi} \mathrm{d} \theta e^{-3.44\left[\left(\frac{D}{r_{0}}\right)\left(\rho \sin \frac{\theta}{2}\right)\right]^{5 / 3}} \cos \Delta \theta,
$$

where $\Delta$ is an integer step in the mode index of $\ell$, and $\rho=$ $2 r / D$ [9].

As shown in Fig. 1, we generate OAM modes by use of a simple forked diffraction grating created using an SLM illuminated by an expanded Gaussian beam produced by a HeNe laser. This results in a helically phased beam, which has a near-Gaussian intensity distribution in the image plane of the SLM. This approach maintains the ratio $D / r_{0}$ independent of the mode index. A particular turbulent phase screen can then be added to this hologram to simulate the presence of atmospheric turbulence. The SLM is then imaged to the $8 \mathrm{~mm}$ diameter input pupil of the OAM mode sorter used to sort the resulting beam into its constituent OAM modes.

The mode sorter uses two refractive elements, which transform OAM states into transverse momentum states $[13,14]$. These elements transform a beam of the form $\exp (i \ell \theta)$, to $\exp (i \ell x / a)$, where $a$ is a scaling parameter. A lens is used to focus these transformed states to discrete spots at a CCD placed in its focal plane. Adjacent, equally sized regions are selected on the CCD image, with each region corresponding to a specific OAM mode. The sum of the measured pixel values in each of these regions is proportional to the power of the beam in each OAM mode. For each input mode, this power is measured across 11 regions corresponding to the range $\ell \in$ $[-5,5]$ and normalized with respect to the signal for input $\ell=0$ with no turbulence applied.

A range of turbulence levels characterized by $D / r_{0} \in$ $\left[10^{-2}, 10^{2}\right]$ were tested, with each data point averaged over 100 realizations. Care was taken to stay within the spatial frequency bandwidth of the SLM, as features smaller than the pixel spacing cannot be represented, setting an upper limit on the $D / r_{0}$ values that can be represented. As predicted by Eq. (2), the crosstalk increases with turbulence. In the mid/high turbulence regime shown, we see good agreement between our measurements and the theory proposed in [9]. In the low turbulence regime, the crosstalk between modes arises from residual crosstalk in our mode sorter, which can be attributed to the diffraction limit $[13,14]$. The weightings of the known input states described by an $N=11$ element column vector $[I]$ are mapped by an $N \times N$ crosstalk matrix onto the measured $N$ element output vector $[O]$ [Eq. (3)]. For the case of zero residual crosstalk, this matrix would correspond to an identity matrix. For finite crosstalk, the coefficients $a-j$ etc. are measured at zero turbulence. Consequently, this matrix is used to predict the measured OAM output spectrum for an input OAM state subject to the atmospheric crosstalk from our theoretical model [Eq. (2)].

$$
\left[\begin{array}{c}
O_{0} \\
O_{1} \\
\vdots \\
O_{N}
\end{array}\right]=\left[\begin{array}{cccc}
1-g & a & \cdots & b \\
c & 1-h & \cdots & d \\
\cdots & \cdots & \cdots & \cdots \\
e & f & \cdots & 1-f
\end{array}\right]\left[\begin{array}{c}
I_{0} \\
I_{1} \\
\vdots \\
I_{N}
\end{array}\right] .
$$

It is seen in Fig. 2 that at high turbulence values $\left(D / r_{0} \gg 1\right)$ the average power is equally spread between all detected modes. It should be noted that we are only considering the proportion of the power detected within the detector regions and not considering the power incident outside these regions.

The theory presented in [9] indicates that the probability of modal crosstalk resulting from atmospheric turbulence is independent of the input mode number. To examine this theory, we studied the effects of turbulence on different OAM modes ranging from

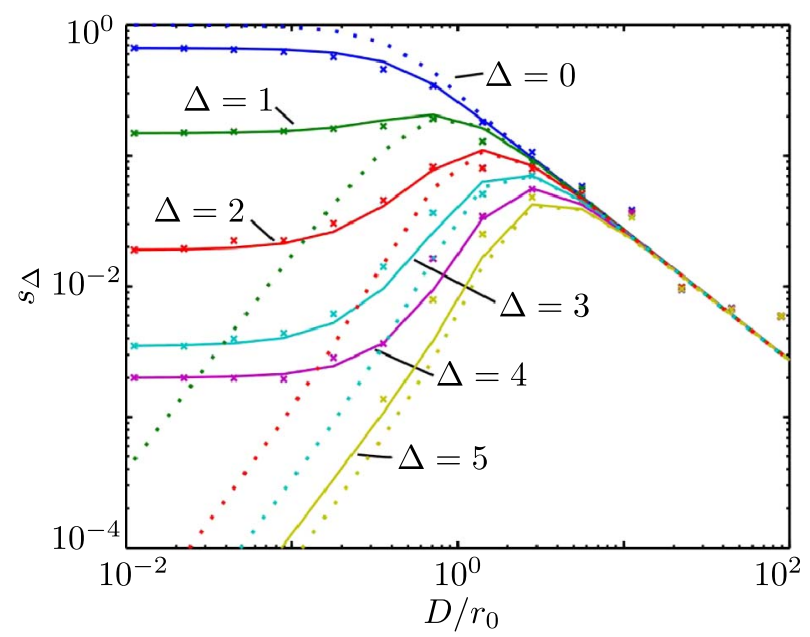

Fig. 2. (Color online) The average power $\left(s_{\Delta}\right)$ in detected mode $\psi_{\Delta}$ is plotted as a function of turbulence strength $\left(D / r_{0}\right)$ for an input mode with $\ell=0$ [see Eq. (2)]. Experimental data (crosses) is coplotted with the theoretical prediction given by Eq. (2) taking into account the inherent crosstalk of the mode sorter (solid lines). The original theory from [9] is also plotted for comparison (dotted lines). 

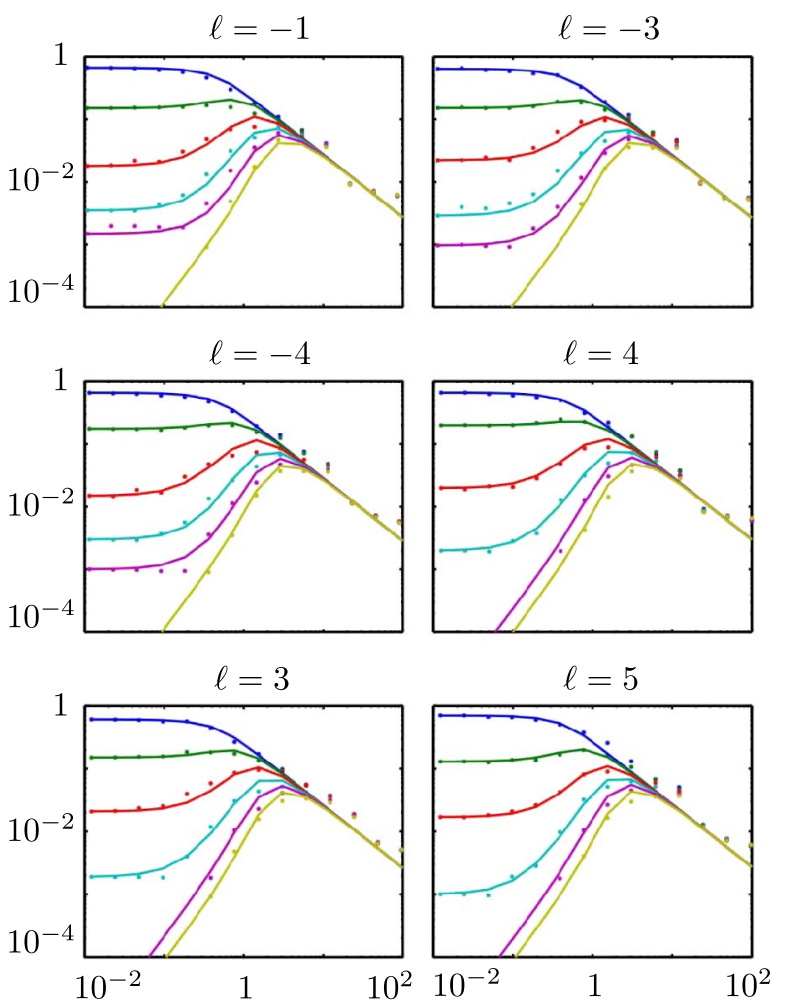

Fig. 3. (Color online) The spread in power resulting from atmospheric turbulence was measured for a range of different propagating OAM modes $\psi_{\ell}$.

$\ell=-5$ to $\ell=+5$. For each of these modes, the same set of turbulent phase screens was applied. The measured crosstalk is shown in Fig. 3. We note that the observed crosstalk is indeed very similar for the entire range of OAM modes that we examined.

In this work we have studied the case where turbulence can be modeled by a thin-phase screen. Such an approach is widely used in astronomy, as when one considers the distance to an astronomical light source, the largest proportion of the turbulence is experienced relatively close to the observer. However, in the case of long distance point-to-point communications on earth, turbulence is characterized more accurately by multiplane turbulence. In such cases, one can expect intensity fluctuations and scintillation effects, and the thin-phase model is insufficient.

Knowledge of the limits that atmospheric turbulence imposes on a free-space communication channel is very important for designing an optical system operating in such an environment. In this letter, we have experimentally characterized the effects of thin-phase turbulence over a range of $\ell=-5$ to $\ell=+5$, and verified that turbulence degrades the mode quality independent of input mode number. This result indicates that a system implementing adaptive optics to reduce the effects of turbulence can operate independently of the communications channel. The experimental data presented also indicates the potential working range of a free-space OAM channel and the expected crosstalk for such a system. We expect that our study will provide useful information for the construction of practical quantum key distribution systems using OAM modes [15].

We acknowledge Daniel Gauthier and Jonathan Leach for helpful discussions. Our work was primarily supported by the Defense Advanced Research Projects Agency (DARPA) InPho program through the U.S. Army Research Office award W911NF-10-1-0395. MPJL was further supported by European collaboration EC FP7 255914, PHORBITECH, and MJP is supported by the Royal Society.

\section{References}

1. J. M. Beckers, Annu. Rev. Astron. Astrophys. 31, 13 (1993).

2. V. I. Tatarski, Wave Propogation in a Turbulent Medium (McGraw-Hill, 1961).

3. D. L. Fried, J. Opt. Soc. Am. 55, 1427 (1965).

4. L. Allen, M. Beijersbergen, R. Spreeuw, and J. Woerdman, Phys. Rev. A 45, 8185 (1992).

5. G. Gibson, J. Courtial, M. J. Padgett, M. Vasnetsov, V. Pas'ko, S. M. Barnett, and S. Franke-Arnold, Opt. Express 12, 5448 (2004).

6. M. Malik, M. O’Sullivan, B. Rodenburg, M. Mirhosseini, J. Leach, M. P. J. Lavery, M. J. Padgett, and R. W. Boyd, Opt. Express 20, 13195 (2012).

7. M. Bourennane, A. Karlsson, and G. Björk, Phys. Rev. A 64, 012306 (2001).

8. C. Paterson, Phys. Rev. Lett. 94 (2005).

9. G. A. Tyler and R. W. Boyd, Opt. Lett. 34, 142 (2009).

10. B. Smith and M. Raymer, Phys. Rev. A 74, 5 (2006).

11. G. Gbur and R. K. Tyson, J. Opt. Soc. Am. A 25, 225 (2008).

12. F. Roux, Phys. Rev. A 83 (2011).

13. G. Berkhout, M. Lavery, J. Courtial, M. Beijersbergen, and M. Padgett, Phys. Rev. Lett. 105 (2010).

14. M. P. J. Lavery, D. J. Robertson, G. C. G. Berkhout, G. D. Love, M. J. Padgett, and J. Courtial, Opt. Express 20, 2110 (2012).

15. R. W. Boyd, A. Jha, M. Malik, C. O'Sullivan, B. Rodenburg, and D. J. Gauthier, Proc. SPIE 7948 79480L (2011). 